\title{
TRADUIRE UN JEU DE PRONOMS OU LA SUBJECTIVITÉ DU TRADUCTEUR REVISITÉE AUTOUR DES TRADUCTIONS POLONAISES D'UN PASSAGE DE MADAME BOVARY
}

\author{
Elżbieta SKIBIŃSKA \\ Université de Wrocław
}

\begin{abstract}
The French pronoun on - whose interpretation depends on various elements of the context - can refer to all humans or to a specific person, identifiable or not; it can be used instead of all personal pronouns to obtain particular stylistic effects. This particularity of on is exploited by writers to create special effects, which can be a challenge for translators. This study examines how Gustave Flaubert used on in a passage of Madame Bovary and the means which were adopted in four Polish translations of the novel; it shows how the chosen solutions (dictated by the grammatical system of Polish language) reduce - each in a different way - the interpretative potential of the analyzed passage.
\end{abstract}

\section{INTRODUCTION}

Les anniversaires peuvent être l'occasion de regarder en arrière et de reprendre des sujets qui nous ont - à un moment ou à un autre - attirés ou absorbés. L'invitation à participer au volume d'hommage à Alicja Kacprzak, consacré au poids des mots, m'a naturellement poussée à revisiter un petit mot qui m'a longuement occupée, le pronom on. La spécificité de son fonctionnement, qui prend sa source dans ce que Fløttum, Jonasson et Norén (2007) appellent «paradoxe de la sémantique » :

D'une part, on établit la présence d'un ou de plusieurs êtres humains comme agissant, percevant, sentant ou subissant une action, bref comme le sujet concerné par une situation décrite. D'autre part, l'identification de 
ce ou ces êtres(s) humain(s) repose sur toute la complexité des indications contextuelles, des connaissances communes et des idées préconçues de l'ontologie du monde des locuteurs. (Fløttum, Jonasson, Norén, $2007: 7)^{1}$

en fait souvent une pierre d'achoppement dans la traduction. En effet, si la présence du pronom dans le texte original (désormais TO) offre un potentiel interprétatif, ou une multitude d'interprétations possibles, le potentiel interprétatif inscrit dans le texte de la traduction (désormais TT), est souvent fortement réduit, voire changé ${ }^{2}$. Ceci peut résulter des différences entre les langues ${ }^{3}$, mais aussi de la lecture propre au traducteur.

C'est à bon escient que j'ai choisi le mot lecture : avant d'être l'auteur du TT proposé à un lecteur de la langue d'arrivée, le traducteur est un lecteur (certes, un lecteur spécial, qui procède à une lecture orientée, menée en vue de la traduction qui s'ensuivra ${ }^{4}$ ) qui actualise le potentiel interprétatif du TO à sa façon et qui l'inscrit dans le TT. Ainsi, entre en jeu ce que j'appelle « la subjectivité du traducteur $»^{5}$. Celle-ci intervient au moment de sa lecture (interprétation) du TO, lorsqu'il mobilise ses compétences linguistiques, stylistiques, rhétoriques, encyclopédiques..., et au moment de l'inscription de cette interprétation dans le TT, lorsqu'il effectue un choix des moyens dont dispose la langue-culture d'arrivée. Aussi chaque traduction du même texte est-t-elle marquée par la subjectivité de son traducteur et offre un potentiel interprétatif différent ${ }^{6}$. En effet, comme le constate Theo Hermans :

The translating subject cannot be elided or eliminated from translations because, as a form of text-production, translating requires the deployment of linguistic means in the host language, and this will involve dimensions

${ }^{1}$ On peut rappeler aussi les mots de Françoise Atlani : « [...] alors que c'est la forme même des pronoms personnels qui permet de comprendre la place des locuteurs dans le procès d'énonciation, c'est l'interprétation [souligné par E.S.] de on qui permet de lui attribuer tel ou tel statut énonciatif » (Atlani, 1984 : 16). Cette propriété du pronom en fait un moyen utilisé dans la fiction romanesque pour créer des effets spéciaux basés sur une ambiguïté voulue : on pouvant avoir aussi bien un référent spécifique que générique, il permet de passer facilement de l'expérience singulière à la généralité (Maingueneau, 2000 : 9).

${ }^{2}$ J'emprunte la notion de potentiel interprétatif à Hewson (2011).

${ }^{3}$ Voir à ce propos Papadima (1997).

${ }^{4}$ Voir Hewson (1995).

${ }^{5}$ Le terme de subjectivité est utilisé comme dérivation du terme sujet, compris comme « être individuel considéré comme support d'une action dans laquelle il laisse sa marque » (Le Nouveau Petit Robert).

${ }^{6}$ Les analyses des retraductions - terrain de prédilection pour observer la subjectivité du traducteur - le prouvent bien. 
other than those of the original. As a result, the translator's utterances are necessarily marked, revealing discursively positioned subject. (Hermans, $2007: 28)$

La « subjectivité revisitée », mentionnée dans le titre, renvoie aux réflexions que j'ai menées il y a quelques années sur le phénomène de la retraduction comme un terrain où se manifeste la subjectivité du traducteur (Skibińska, 2007). Je me suis concentrée à l'époque sur les attitudes opposées des traducteurs face à la spécificité culturelle de Pan Tadeusz d'Adam Mickiewicz. Dans les lignes qui suivent, je vais me pencher sur des éléments linguistiques et, plus précisément, sur un emploi particulier du pronom on dans un bref passage de Madame Bovary. Quatre traductions polonaises du roman proposent des solutions différentes du problème résultant du recours que fait Flaubert à ce pronom. Mon objectif n'est pas d'apporter un jugement de valeur sur ces solutions, mais de montrer comment, par le choix effectué dans l'inventaire des possibilités dont dispose le polonais, se manifeste l'interprétation du traducteur, et comment il façonne, par ses choix, le potentiel interprétatif du TT. Dans cette démarche, je suis de l'avis exprimé par Lance Hewson :

[Translation criticism] involves an interpretative act whereby the basis of the value judgement is explicitly spelled out. Translation criticism attemps to set out the interpretative potential of a translation seen in the light of an established interpretative framework whose origin lies in the source text. It thus goes beyond both implicit (and indeed unsubstantiated) judgements, and those approaches that seek to pinpoint specific weaknesses of a particular translation (or a set of translations). Translation criticism is evaluative, in that as it explores a translation's interpretative potential, it looks at degrees of similarity to or divergence from the source text's perceived interpretative potential. (Hewson, $2011: 6-7$ )

\section{LeCTURe(s) DE L'ORIGINAL}

Le passage de Madame Bovary qui m'intéresse se situe près du milieu du roman, à la fin du chapitre 7 de la deuxième partie, lorsque Rodolphe Boulanger fait son apparition dans l'histoire. Propriétaire depuis peu du château de la Huchette près d'Yonville, riche, vivant " en garçon » (p. 119), il amène chez Charles Bovary un domestique souffrant. À cette occasion, il voit pour la première fois Emma, dont l'apparence éveille son intérêt : « de tempérament brutal et d'intelligence perspicace, ayant d'ailleurs beaucoup fréquenté les femmes 
et s'y connaissant bien » (p. 121-122), il reconnaît en elle un éventuel objet de séduction. Sur le chemin de retour à son château, il songe au couple dont il vient de faire la connaissance :

(1) - Elle est fort gentille ! se disait-il ; elle est fort gentille, cette femme du médecin! De belles dents, les yeux noirs, le pied coquet, et de la tournure comme une Parisienne. D'où diable sort-elle ? Où donc l'a-t-il trouvée, ce gros garçon-là ? (p. 121)

Dès ce passage se voit la façon dont Rodolphe perçoit sa future maîtresse : Emma (comme d'autres femmes probablement) est pour lui une somme de traits physiques agréables, un objet de plaisir, et non pas une personne. Cette perception de la femme est renforcée dans la suite de son monologue :

(2) - Je le crois très bête. Elle en est fatiguée sans doute. Il porte des ongles sales et une barbe de trois jours. Tandis qu'il trottine à ses malades, elle reste à ravauder des chaussettes. Et on s'ennuie ! on voudrait habiter la ville, danser la polka tous les soirs! Pauvre petite femme ! Ça bâille après l'amour, comme une carpe après l'eau sur une table de cuisine. Avec trois mots de galanterie, cela vous adorerait, j'en suis sûr ! ce serait tendre ! charmant !... Oui, mais comment s'en débarrasser ensuite ? (p. 122)

Les deux passages sont des occurrences de discours rapporté, reproduisant les pensées de Rodolphe. Le thème en est Emma, désignée dès la première phrase par le pronom elle, qui ouvre le premier passage. Dans la deuxième phrase (répétition de la première), le pronom est repris, en position finale, par le syntagme cette femme du médecin qui en précise la valeur référentielle. Cette dislocation a une double fonction. Elle souligne l'importance de l'objet de sa réflexion, tout en le « définissant »: Emma n'a d'existence que comme femme du médecin. Dans la phrase suivante, elle est réduite à quelques attraits de son physique, énumérés dans une série de syntagmes nominaux. Dans les deux phrases suivantes, traduisant l'étonnement de Rodolphe devant la beauté inattendue dans la petite ville de province, Emma est d'abord saisie comme un être indépendant (désigné par le pronom elle, sujet du verbe sortir intransitif), pour, ensuite, devenir à nouveau un « objet » appartenant à son mari (désigné par le pronom la, complément d'objet du verbe trouver). Le syntagme ce gros garçon-là (disloqué lui aussi, reprenant le pronom $i l$ ) annonce le mépris de Rodolphe pour Charles, explicite dans le deuxième passage (Je le crois très bête; Il porte des ongles sales et une barbe de trois jours ; il trottine à ses malades). 
Il me semble significatif que deux fois seulement les époux Bovary soient désignés par un syntagme nominal, alors qu'ils continuent à être le thème des pensées de Rodolphe, d'abord tous les deux, ensuite Emma toute seule. Dans le deuxième passage, à part l'exclamation Pauvre petite femme !, c'est toujours par les pronoms qu'ils sont désignés. Et c'est précisément le jeu des pronoms renvoyant à Emma qui fonde le potentiel interprétatif du passage (2) que je vais étudier ${ }^{7}$.

Dans la première phrase où il est question d'Emma, celle-ci est désignée par le pronom elle; cette phrase se situe entre deux autres dont le thème est Charles : désigné d'abord par le, complément d'objet, il suscite une appréciation négative de ses (supposées) capacités intellectuelles. Ensuite, il est le sujet d'une phrase qui apporte encore une caractérisation négative de son physique. Les deux fournissent des arguments qui fondent la conviction ou conclusion (exprimée par sans doute) qu'Emma peut être une conquête facile (son mari ne l'attire plus). Cette conviction est renforcée dans la suite du raisonnement de Rodolphe : Emma, la plupart du temps seule et occupée par des tâches quotidiennes sans intérêt, doit rêver d'un changement, d'un divertissement. Dans les deux phrases qui présentent son (supposé) état d'esprit, elle est désignée par le pronom on, dont la présence offre diverses possibilités d'interprétation : il peut être considéré comme (a) un substitut anaphorique de elle, avec une valeur ajoutée, celle d'une condescendance de la part de Rodolphe à l'égard de la "pauvre petite femme »; il peut être lu comme (b) un moyen de donner, pour un moment, la parole à Emma elle-même, le pronom serait alors interprété comme je, renvoyant à la femme ${ }^{8}$; ou encore (c) on serait utilisé à la place d'une $2^{\mathrm{e}}$ personne $(t u$, vous $)$, comme si Rodolphe s'adressait à Emma, avec un ton de commisération'. Dans tous les cas, il s'agit de la situation d'Emma telle qu'elle est imaginée par Rodolphe qui met en oeuvre sa connaissance des femmes, ou un stéréotype : Emma est comme toutes les femmes qui s'ennuient car elles sont fatiguées par leur mari et leur quotidien.

\footnotetext{
${ }^{7}$ J'ai commenté ce passage dans Skibińska, 2006 (p. 159). Dans la suite, je vais en approfondir l'examen pour en présenter le potentiel interprétatif (en en offrant ma lecture subjective).

${ }^{8}$ Telle est la lecture que proposent Fløttum, Jonasson et Norén (op. cit., p. 60), en soulignant que l'emploi de on au lieu de elle permet au narrateur de brouiller « la frontière non seulement entre la conscience de Rodolphe et celle d'Emma, le premier pénétrant dans le monde imaginaire de la dernière, mais aussi celle entre son propre univers et celui des personnages ». Remarquons toutefois que si, pour une fois, la voix d'Emma se laisse entendre, elle est cependant " mimée » ou " citée » par Rodolphe.

${ }^{9}$ Dans les trois lectures, il serait question d'un emploi stylistique tel que le définit Charles Muller : on peut être utilisé à la place de chacun des pronoms personnels, et permet alors d'obtenir des effets stylistiques particuliers, telle « une intention affective : ironie, mépris, tendresse, euphémisme, discrétion affectée, bonhomie, etc. » (Muller, $1970: 51-52$ ).
} 
L'exclamation Pauvre petite femme ! qui suit constitue une sorte de commentaire que fait Rodolphe de la situation d'Emma : aussi bien l'adjectif pauvre, en antéposition, que petite - hypocoristique conventionnel - servent à la présenter comme inspirant de la pitié, de la commisération, mais aussi du mépris ou du dédain ; c'est la connaissance d'autres situations semblables qui permet à Rodolphe de reconnaître un phénomène récurrent. Emma perd ainsi son unicité.

La présence du pronom on a cependant encore un autre effet : il sert de « transit» entre elle et ça dans la série de pronoms elle - on - ça, cela, ce qui marque la réification progressive d'Emma dans la pensée de Rodolphe. En effet, dans les phrases successives, elle est désignée d'abord par le pronom personnel elle, qui renvoie à elle en tant que personne (femme) spécifique, ensuite par le pronom dit impersonnel on qui fait d'elle une parmi des semblables et, enfin, par le pronom démonstratif neutre ça (suivi par cela et ce) qui peut être lu comme permettant de " décatégoriser péjorativement un référent en lui refusant sa dénomination usuelle » (Riegel, Pellat, Rioul, 1994 : 206) ou encore - comme désignant une personne, avec une valeur affective, celle de mépris, en l'occurrence. Cette suite de pronoms fait ainsi d'Emma un objet qui servira à donner du plaisir pendant un certain temps, avant le moment où il faudra " s'en débarrasser ». On peut remarquer ici que le pronom en a lui aussi une double valeur : si " son usage s'étend aujourd'hui aux animés » (Riegel, Pellat, Rioul, ibid., p. 201), il est destiné principalement à indiquer les non animés.

De telles considérations mènent naturellement Rodolphe à conclure : « Oh ! je l'aurai !» (p. 122).

Le passage étudié n'est qu'un exemple de l'usage que fait Flaubert des pronoms, qui n'a rien d'un hasard chez cet auteur bien connu pour son travail sur le style, et notamment sur la recherche de concision ${ }^{10}$. Il est d'autant plus intéressant de voir les propositions de lecture inscrites dans les traductions.

\section{LECTURE DES TRADUCTIONS}

La première version polonaise du passage qui nous intéresse (1878) révèle les difficultés que tout traducteur devra affronter, puisqu'elles résultent des différences des systèmes linguistiques en jeu. La première concerne l'inventaire des pronoms (personnels) et, plus particulièrement, l'existence, en polonais, de trois genres face à deux en français et l'absence d'un pronom équivalent de on. La deuxième est de nature syntaxique : si, en français, le sujet du verbe est

\footnotetext{
${ }^{10}$ Voir p. ex. les analyses présentées dans Fløttum, Jonasson, Norén (op. cit., p. 54 sq.).
} 
toujours présent dans la phrase (par ex. sous la forme d'un pronom personnel sujet), en polonais il est indiqué par la terminaison du verbe (on parle d'un podmiot domyślny - « sujet par défaut »), tandis que la présence du pronom personnel sujet a un caractère marqué.

Dans les traductions citées, toutes les occurrences de la disparition du pronom sujet dans la traduction sont marquées par le symbole Ø.

(TT1) Ø Wydał mi się bardzo głupim. Ø Musi być nim znudzona. Ø Ma brudne paznokcie i brodę od trzech dni nie goloną. Podczas gdy on odwiedza swych chorych, ona siedzi w domu i ceruje jego skarpetki. I Ø nudzi się, biedactwo! Chciałoby się to mieszkać w mieście, tańczyć w wieczór poleczkę! Biedna kobiecina! Tęskni to za miłościa, jak karp za wodą gdy leży na kuchennym stole. Żeby temu trzy grzeczności powiedzieć, toby się $\varnothing$ zakochała po uszy! ani wątpić! Byłoby to czułe, rozkoszne... tak, ale jak się tego pozbyć w danym czasie? (p. 219-220)

On remarque d'emblée les deux pronoms sujets gardés dans la phrase Podczas gdy on odwiedza swych chorych, ona siedzi $w$ domu i ceruje jego skarpetki. Leur présence permet de contraster les activités de Charles (à l'extérieur, sans la valorisation négative de l'original : dans TT1, il 'visite ses malades') et la monotonie de la vie d'Emma (qui reste à la maison à ravauder les chaussettes de son mari). L'ambiguïté du on, sujet de on s'ennuie, disparaît : dans $n u d z i$ się, biedactwo c'est à Emma que renvoie, par anaphore, le verbe à la $3^{\mathrm{e}}$ pers. sing. ; le substantif biedactwo ('pauvrette'), sujet mis en position finale, exprime l'apitoiement ou la commisération de la part de Rodolphe, mais aussi, par la forme du genre neutre, une sorte de diminution de l'importance d'Emma en tant que femme ou personne. Le neutre apparaît aussi dans la traduction de on voudrait, rendu par chciatoby się : forme à sens impersonnel qui, comme on, peut se substituer à toutes les personnes et introduire une valeur stylistique affective ; comme dans le TO, le recours à ce moyen apporte, dans TT1, un affaiblissement de la saisie d'Emma comme personne ${ }^{11}$. Si dans le commentaire apitoyé Biedna kobiecina ('pauvre femme') elle l'est encore, dans les phrases qui suivent, où elle est désignée par le pronom neutre to ('cela'), soit sujet (deux occurrences), soit complément d'objet (deux occurrences), elle se voit priver de son statut de personne. On peut constater que, malgré les différences grammaticales, le potentiel interprétatif du TT1 ne diverge pas considérablement de celui du TO : après les premières phrases où Emma est saisie comme une personne, à la suite du commentaire Biedna kobiecina, elle devient un objet, désigné par to.

${ }^{11}$ Remarquons la présence de la particule to, qui ajoute une valeur expressive à la phrase. 
L'auteur de la deuxième traduction de Madame Bovary (1912) précise dans sa préface que la version antérieure, non dépourvue de qualités, ne satisfait plus aux nouvelles exigences des lecteurs ; la nouvelle traduction garde cependant des passages de la précédente. C'est partiellement le cas de celui qui nous intéresse :

(TT2) Ø Wydał mi się bardzo głupim. Ø Musi być nim znudzona. $\varnothing$ Miał brudne paznokcie i brodę od trzech dni nie goloną. Podczas gdy on odwiedza swych chorych, ona siedzi w domu i ceruje jego skarpetki. Ø Nudzi się, biedactwo! Chciałoby się mieszkać w mieście, tańczyć co wieczór poleczkę! Biedna kobieta! Tęskni za miłością jak karp za wodą gdy leży na kuchennym stole. Powiedzieć jej trzy grzeczne słówka, toby się Ø zakochała po uszy! jestem pewny! Byłoby to czułe, rozkoszne... tak, ale jak się tego pozbyć w odpowiednim czasie? (p. 164-165)

On remarque que les phrases à sujet on n'ont pas subi de modifications significatives par rapport à TT1. Les divergences commencent à partir de la phrasecommentaire Pauvre petite femme! rendue par Biedna kobieta. Avec la forme de base du substantif kobieta, la charge expressive (commisération) est plus réduite, elle est portée par le seul adjectif biedna. Mais la différence principale - aussi bien par rapport à TT1 que TO - est apportée dans les deux phrases suivantes par l'emploi des formes du verbe à la $3^{\text {e }}$ pers. sing. (tęskni, zakochata się) et du pronom personnel jej ('lui'), qui, dans une chaîne anaphorique, renvoient à Emma, toujours en tant que femme (donc saisie comme une personne). Ce n'est que dans la dernière phrase qu'elle perd ce caractère et devient un objet (désigné par to).

La troisième traduction (1955):

(TT3) Ø Jest pewno bardzo głupi. Ø Musi być nim znudzona. Pan doktor ma brudne paznokcie i brodę od trzech dni nie golona. Gdy $\varnothing$ drepce do chorych, ona siedzi w domu i ceruje skarpetki. I Ø nudzi się, biedactwo. Chciałoby to zamieszkać w mieście, tańczyć polkę co wieczór! Biedna kobietka! Tęskni to za miłościa, jak karp na kuchennym stole za woda. Pokochałoby to za parę tkliwych słówek, jestem pewien! Byłoby czułe, urocze!... Tak, ale jak się jej potem pozbyć? (p. 120-121)

se démarque des précédentes par le traitement de la deuxième phrase avec on: le sujet de la phrase polonaise est le pronom neutre to, coréférent de biedactwo (genre neutre) de la phrase précédente. Le statut d'Emma est ainsi réduit à celui d'un être immature (comme un enfant), voire d'un objet. Si dans le commentaire Biedna kobietka elle recouvre encore son statut de femme, c'est quand même 
de façon dévalorisante (la personne désignée par le diminutif kobietka, 'petite femme', ne semble pas un être humain à caractère " complet », elle manque de sérieux ou de maturité). Dans la suite, elle est indiquée de façon anaphorique par une série de to et de verbes à la $3^{\mathrm{e}}$ pers. sing. neutre. Dans la dernière phrase, Emma, désignée par le pronom $j e j$, « redevient » une femme.

La traduction la plus récente $(2005)^{12}$ propose une lecture bien différente du passage :

(TT4) Ø Wygląda mi na durnia. Na pewno już jej Ø obrzydł. Ø Ma brudne paznokcie i trzydniowy zarost. Kiedy $\varnothing$ gania po swoich chorych, ona w domu ceruje skarpetki. Nudzimy się strasznie! Chcielibyśmy mieszkać w mieście i co wieczór tańczyć polkę! Biedactwo! Toż to wzdycha za miłościąjak karp za wodą na kuchennym stole. Kilka komplementów i toto się zakocha, słowo daję! Będzie czułe, rozkoszne!... No tak, ale jak jej się potem pozbyć? (p. 128)

Ce qui frappe d'emblée, c'est l'économie de l'expression qui répond à la recherche de la concision qui caractérise la démarche de Flaubert. Dans le TT4, on observe l'absence de tout substantif sujet. Le thème des réflexions de Rodolphe - Charles et Emma - est posé et il n'y a pas lieu de le rappeler ; chacun des époux est désigné par la forme du verbe ou par le pronom qui convient. C'est notamment le cas des premières phrases dont le thème est Charles ; il l'est aussi dans la subordonnée Kiedy gania po swoich chorych [...]. Le pronom ona - sujet de la principale (ona $w$ domu ceruje skarpetki) sert à accentuer le changement du thème (les pensées de Rodolphe passent de Charles à Emma) et le contraste entre l'activité de l'un et la vie monotone de l'autre des époux. C'est toujours à Emma que se rapportent les deux phrases suivantes (Nudzimy się strasznie! Chcielibyśmy mieszkać w mieście...), mais l'élément grammatical qui renvoie à Emma (« le sujet par défaut ») est la terminaison verbale, celle de la $1^{\text {ère }}$ pers. du pluriel. Nous sommes ainsi face à un exemple, bien intéressant, d'énallage de la personne. En effet, la $1^{\text {ère }}$ pers. du pluriel peut être utilisée pour s'adresser à un interlocuteur à la place de la $2^{\mathrm{e}}$ personne, avec un effet hypocoristique (comme dans les paroles adressées à un enfant : Pójdziemy spać? 'nous allons dormir ?', ou à un malade : Dobrze się dziś czujemy? 'nous allons bien aujourd'hui ?'). Si Emma, absente, ne peut pas être l'interlocutrice de Rodolphe, il ressort néanmoins de ces formes, qui doivent rendre l'effet dû au pronom on du TO, la condescendance avec laquelle elle est perçue par Rodolphe. Cet effet est renforcé par le mot biedactwo (traduisant le

${ }^{12}$ Dans la version corrigée de cette traduction publiée en 2014, le passage analysé n'a pas été modifié. 
commentaire Pauvre petite femme!). Avec les pronoms to et toto, la mésestime de Rodolphe à l'égard d'Emma devient encore plus profonde : elle n'est que l'objet de calcul d'un éventuel amusement.

\section{Pour conclure}

Quatre traducteurs polonais se sont trouvés devant le défi lancé par un « petit mot » de deux lettres, mais d'un grand poids interprétatif. Tous ont puisé dans les ressources offertes par le polonais. Chacun l'a fait à sa manière et, inévitablement, avec un résultat différent. La réification progressive d'Emma visible dans les considérations de Rodolphe se retrouve dans les traductions polonaises, quoiqu'à des degrés variés. Le recours à une forme impersonnelle ou l'énallage de la personne permettent de rendre, ne serait-ce qu'en partie, le potentiel interprétatif obtenu dans le TT par l'emploi du pronom on (et, dans la suite, de la série ça, cela, ce). C'est la reproduction de l'effet « esthétique » de on, outil de concision, qui semble le problème le plus difficile à résoudre.

\section{Textes analysés}

TO : FLAUBERT, Gustave (1961 [1857]) Madame Bovary. Moeurs de province (Introduction, notes et relevés de variantes par Édouard Maynial), Paris, Éditions Garnier Frères.

TT1 : FLAUBERT, Gustave (1878), Pani Bovary (trad. Ludwika Kaczyńska), Warszawa, Redakcya Przeglądu Tygodniowego.

TT2 : FLAUBERT, Gustave (1912), Pani Bovary (trad. Alfred Iwieński), Warszawa, Gebethner i Wof, Ultima Thule.

TT3 : FLAUBERT, Gustave (1955), Pani Bovary (trad. Aniela Micińska), Warszawa, PIW.

TT4 : FLAUBERT, Gustave (2005), Pani Bovary (trad. Ryszard Engelking), Gdańsk, Słowo/Obraz Terytoria.

\section{Références bibliographiques}

ATLANI, Françoise (1984), «On l'illusionniste », in La langue au ras du texte (A. Grésillon, J.-L. Lebrave éd.), Lille, Presses Universitaires du Septentrion, pp. 13-27.

FLØTTUM, Kjersti, JONASSON, Kerstin, NORÉN, Coco (2007), ON : pronom à facettes, Bruxelles, Éditions Duculot.

HERMANS, Theo (2007), The Conference of the Tongues, Manchester, St Jerome.

HEWSON, Lance (1995), « Images du lecteur », Palimpsestes, n 9, pp. 151-164.

HEWSON, Lance (2011), An Approach to Translation Criticism: Emma and Madame Bovary in Translation, Amsterdam/Philadelphia, Benjamins. 
MAINGUENEAU, Dominique (2000), Éléments de linguistique pour le texte littéraire, $3^{e}$ éd., Paris, Nathan.

MULLER, Charles (1970), « Sur les emplois personnels de l'indéfini on », Revue de Linguistique Romane, t. 34, pp. 48-55.

PAPADIMA Maria (1997), « Compte rendu d'un échec. La traduction du on dans les Éblouissements de Pierre Mertens », Metafrassi, n 3, pp. 147-152.

RIEGEL, Martin, PELLAT, Jean-Christophe, RIOUL, René (1994), Grammaire méthodique du français, Paris, PUF.

ROBERT, Paul, REY-DEBOVE, Josette, REY, Alain (1993), Le Nouveau Petit Robert, Paris, Dictionnaires Le Robert.

SKIBIŃSKA, Elżbieta (2006), « Dépersonnalisation et anonymat au service des idées reçues : observations sur le pronom on dans Madame Bovary ", Romanica Wratislaviensia $\mathrm{n}^{\circ}$ LIII, pp. 151-160.

SKIBIŃSKA, Elżbieta (2007), « La retraduction, manifestation de la subjectivité du traducteur », Doletiana. Revista de traducció, literatura i arts, $\mathrm{n}^{\circ} 1$ (« Subjecte i traducció »), www.fti.uab. cat/doletiana (dernière consultation : le 15.11.2017). 